\title{
Expresión y actividad de posibles polimorfismos provenientes de individuos normales en la proteína de 67 kd del sistema NADPH oxidasa utilizando el sistema $\operatorname{COS}^{\text {phox }}$
}

\begin{abstract}
Andrés Augusto Arias ${ }^{1}$, Mary C. Dinauer ${ }^{2}$, Jiabin Ding ${ }^{3}$, Juan David Matute ${ }^{1}$, Pablo Javier Patiño ${ }^{1}$
${ }^{1}$ Grupo de Inmunodeficiencias Primarias, Corporación Biogénesis, Universidad de Antioquia, Medellín, Antioquia, Colombia.

2 Department of Pediatrics, Hematology/Oncology, Wells Center for Pediatric Research, Indiana University School of Medicine, Indianapolis, IN, U.S.A.

${ }^{3}$ Department of Immunology, Genentech Inc., South San Francisco, CA, U.S.A.

El sistema NADPH oxidasa de las células fagocíticas cumple una función importante durante la respuesta antimicrobiana del organismo. La activación de este sistema está precedida por la translocación de las proteínas citosólicas $p 67^{\text {phox }}, \mathrm{p} 47^{\text {phox }}$ y $\mathrm{p} 40^{\text {phox }}$ hacia la membrana para ponerse en contacto con el flavocitocromo $\mathrm{b}_{558}$, lo que induce la generación del anión superóxido, un precursor de agentes microbicidas oxidantes. El presente trabajo presenta un análisis funcional del sistema NADPH oxidasa basado en los hallazgos de polimorfismos encontrados en el gen de $p 67^{\text {phox }}$ de individuos sanos. Para esto se generaron mutaciones en el cADN que codifica la p67 ${ }^{\text {phox }}$ y se expresaron en el sistema de células COS ${ }^{\text {phox }}$. Los datos

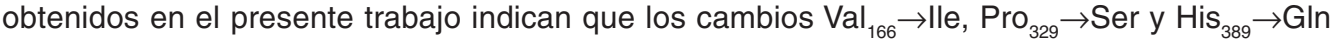
no generan alteraciones en el funcionamiento de la p67 phox cuando su función se analizó en el sistema transgénico basado en células COS-7. Por lo tanto, estos polimorfismos no generan ningún riesgo genético de producir deficiencias en la activación del sistema NADPH oxidasa. Además, se demuestra que el modelo de células COS $^{\text {phox }}$ representa un nuevo sistema celular, fácilmente transfectable que permite estudiar la función del sistema NADPH oxidasa de las células fagocíticas y sus particularidades genéticas. Finalmente, los hallazgos con estos polimorfismos nos permiten avanzar en el conocimiento sobre los mecanismos moleculares involucrados en la activación del sistema NADPH oxidasa células fagocíticas.
\end{abstract}

Palabras clave: NADPH oxidasa, p67 ${ }^{\text {phox }}$, COS-phox cells, polimorfismos.

Expression and activity of polymorphisms in the 67-kDa protein of the NADPH oxidase system

The NADPH oxidase system plays a central role in the antimicrobial activity of phagocytes. This system is initiated by the translocation of cytosolic proteins $p 67^{\text {phox }}, \mathrm{p} 47^{\text {phox }}$ and $\mathrm{p} 40^{\text {phox }}$ to be in close contact with membrane flavocytochrome $b_{558}$. This event begins the electron transfer from cytosolic NADPH to molecular oxygen to produce superoxide anions. Herein, a functional analysis is presented of p67phox polymorphisms identified from healthy humans. Mutations were generated in the p67 $7^{\text {phox }} \mathrm{CDNA}$ by site-directed mutagenesis and then transiently expressed in COS7 cells that also expressed gp91 phox, p22 phox, and p47 phox from stable transgenes. The changes Val166lle, Pro329Ser and His389GIn correspond to possible polymorphisms identified in healthy individuals revealed a functional activity similar to COS ${ }^{\text {phox }}$ cells transiently transfected with WT p67 ${ }^{\text {phox; }}$, therefore, these modifications are not associated with genetic deficiencies in NADPH oxidase. In conclusion, the COSphox system represents an easily transfectable model for analysis of NADPH oxidase function in intact cells. The analysis of mutant derivatives of p67 $7^{\text {phox }}$ provides insight into molecular mechanisms by which this subunit regulates the NADPH oxidase.

Key words: NADPH oxidase, p67 phox, COS-phox cells, polymorphisms. 
Los neutrófilos y otros fagocitos son las únicas células capaces de generar durante un fenómeno conocido como 'explosión respiratoria' grandes cantidades de especies reactivas de oxígeno en respuesta a una gran variedad de estímulos inflamatorios, tanto solubles como particulados. Durante esta explosión respiratoria se transfieren electrones desde el NADPH citosólico al oxígeno molecular que se encuentra en la vacuola fagocítica o en el espacio extracelular, generando así anión superóxido $\left(\mathrm{O}_{2}{ }^{-}\right)(1)$; este fenómeno depende del sistema NADPH oxidasa u oxidasa de las células fagocíticas.

El sistema oxidasa está formado por cinco componentes principales: gp91 $1^{\text {phox }}$ (phox por phagocyte oxidase), p22 $2^{\text {phox }}, \mathrm{p} 67^{\text {phox }}, \mathrm{p} 47^{\text {phox }} \mathrm{y}$ $\mathrm{p} 40^{\text {phox }}$. La interacción de las proteínas gp91 $1^{\text {phox }} \mathrm{y}$ la p22 $2^{\text {phox }}$ forma un heterodímero transmembranal conocido como flavocitocromo $b_{558}$, el cual contiene los elementos necesarios para el transporte de electrones (2-4). Durante la activación del sistema oxidasa, las subunidades citoplasmáticas $\mathrm{p} 40^{\text {phox }}$, $\mathrm{p} 47^{\text {phox }}$ y $\mathrm{p} 67^{\text {phox }}$ se desplazan en bloque para unirse al flavocitocromo $b$ en la membrana plasmática. Una vez ocurre la interacción, el NADPH es oxidado a NADP+, los electrones son transportados al grupo FAD presente en el flavocitocromo $b_{555}$, luego a sus dos grupos heme $y$, finalmente, al $\mathrm{O}_{2} \cdot-(5,6)$. Además de las proteínas phox, la activación de este sistema también requiere de la proteína unidora de GTP Rac con actividad GTPasa (Rac1 o Rac2) (7).

Aunque dichas especies reactivas de oxígeno son importantes en la respuesta microbicida, cuando se generan en cantidades elevadas pueden producir daño en los tejidos del hospedero, lo cual ha llevado a establecer su participación en la patogénesis de enfermedades asociadas con inflamación crónica y aguda tales como la artritis reumatoidea (8), el síndrome de dificultad respiratoria del adulto (9) y el choque séptico (10).

\footnotetext{
Correspondencia:

Andrés Augusto Arias, Sede de Investigaciones Universitarias (SIU), Calle 62 \#52-59, Oficina 530, Universidad de Antioquia, Medellín, Antioquia, Colombia. Teléfono: (574) 210 6471; fax: (574) 5106047 aarias@quimbaya.udea.edu.co
}

Recibido: 17/09/03; aceptado: 08/07/04
Por lo tanto, su producción debe estar altamente regulada para asegurar que se produzcan en el sitio adecuado y en el momento en que sean requeridos $(1,5)$.

La importancia biológica del sistema NADPH oxidasa se ha demostrado gracias a la enfermedad granulomatosa crónica, un trastorno hereditario del sistema inmune caracterizado por la ausencia o, en algunas ocasiones, por la disminución de la producción de $\mathrm{O}_{2} \cdots$ El anión superóxido y sus derivados tóxicos (peróxido de hidrógeno, ácido hipocloroso y radical hidroxilo) (6,11-14) son necesarios para destruir algunos microorganismos invasores; por tanto, los pacientes con enfermedad granulomatosa crónica sufren infecciones recurrentes y algunas veces fatales por bacterias y por ciertos hongos $(15,16)$.

La forma más común de enfermedad granulomatosa crónica es la ligada al cromosoma $X$, que se presenta en $\sim 65 \%$ de todos los casos y es causada por una mutación en el gen $C Y B B$ que codifica para la gp91 ${ }^{\text {phox }}(17,18)$. Los casos restantes de enfermedad granulomatosa crónica se heredan de forma autosómica recesiva y son causadas por defectos en los genes CYA (p22 ${ }^{\text {phox }}$ ), NCF-1(p47 phox y NCF-2 (p67 phox). La enfermedad granulomatosa crónica por deficiencia de p67 $7^{\text {phox }}$ es una forma rara de la enfermedad y se presenta en $6 \%$, aproximadamente, de los pacientes.

Hasta la fecha se han descrito aproximadamente quince mutaciones en el gen NCF-2 $y$ todas ellas dan como resultado una ausencia total del producto proteico (19-23). De la misma manera, se han reportado varios candidatos para polimorfismos en el gen NCF-2 $(24,25)$.

La p67 $7^{\text {phox }}$ es una proteína levemente ácida (pl 6) con 526 aminoácidos y una masa de 60,9 kd, la cual está codificada por el gen NCF2 que está ubicado en el cromosoma 1q25; éste tiene una longitud de $40 \mathrm{~kb}$ y contiene 16 exones que generan un mARN de 2,4 kb (26). En esta proteína se han descrito diferentes tipos de dominios estructurales que tienen un papel importante para su función. En la región amino terminal posee cuatro dominios conocidos como repeticiones tetratricopeptídicas (TPR); tres de ellos se encuentran continuos y el cuarto se encuentra 
separado de los tres primeros por una secuencia de 16 aminoácidos (27-32). Juntos, estos cuatro dominios hacen de la región amino terminal de p67 $7^{\text {phox }}$ un dominio funcional (residuos 1-246) que permite la activación del sistema oxidasa en sistemas libres de células (33) y se une a la proteína Rac $(28,29,31,32)$.

Una de las características más importantes de las proteínas citosólicas del sistema NADPH oxidasa es la presencia de los denominados dominios de homología 3 con Src (SH3) que se encuentran en una gran variedad de proteínas de señalización intracelular que se desplazan entre el citosol y la membrana o en complejos relacionados con el citoesqueleto $(1,34,35)$. Los dominios $\mathrm{SH} 3$ se unen en forma no covalente a regiones ricas en prolinas que se encuentran ubicadas en las proteínas blanco. Tanto la p67 phox como la p47 phox contienen dos dominios SH3 y una región rica en prolinas; por su parte, la proteína p40 ${ }^{\text {phox }}$ posee un dominio SH3 (36-38). La presencia de estos dominios en $\mathrm{p} 67^{\text {phox }}$ le permite interactuar y formar complejos con $\mathrm{p} 47^{\text {phox }}$ y $\mathrm{p} 40^{\text {phox }}$.

El papel exacto de $p 67^{\text {phox }}$ y las demás proteínas phox citosólicas es desconocido; sin embargo, es claro que $\mathrm{p} 67^{\text {phox }}$ es absolutamente requerida para la generación del anión superóxido en células intactas y en sistemas libres de células.

En el presente trabajo se presenta una aproximación al estudio del sistema NADPH oxidasa basada en los hallazgos del análisis de tres mutaciones distintas que se habían identificado previamente en el gen de $p 67^{\text {phox }}$ de individuos sanos (25). Debido a que la enfermedad granulomatosa crónica por deficiencia de p67 phox es un rasgo autosómico recesivo y que dichos cambios se detectaron en individuos sanos heterocigóticos, no era posible saber si estos individuos eran portadores de mutaciones que alteraban la expresión de la p67 $7^{\text {phox }}$ o simplemente correspondían a polimorfismos de dicha proteína.

Con el objetivo de determinar el efecto de estas mutaciones sobre el sistema NADPH oxidasa, se generaron mutaciones en el cADN de $p 67^{\text {phox }}$ y se expresaron en el sistema de células COS $^{\text {phox }}$, un modelo recientemente desarrollado basado en células COS-7, originarias del riñón del mono verde africano (39). Las células COS91/22/47/67 (COS ${ }^{\text {phox }}$ ) exhiben un alto nivel de actividad del sistema NADPH oxidasa cuando son activadas con acetato de forbol miristato (phorbol myristate acetate, PMA) o ácido araquidónico, que son dos estimulantes solubles comúnmente usados para activar el sistema NADPH oxidasa de los neutrófilos. Estas células son fácilmente transfectables y expresan constitutivamente la proteína Rac1.

\section{Materiales y métodos \\ Obtención del cADN de p67phox $y$ características del vector pBfox67}

El cADN de p67 $7^{\text {phox }}$ utilizado se encontraba clonado en el vector pVL1393 (Pharmingen, San Diego, CA) en los sitios Xbal y Pstl. El diseño de los oligonucleótidos, el mapa de restricción y la secuencia de nucleótidos después de la reacción de obtención de secuencias se basó en la secuencia del ARNm de p67 phox publicada en el GenBank (Accession \# NM_000433). El tamaño total del inserto era de 2,4 kb constituido por una secuencia de 1581 pb que codifica para $67^{\text {phox }} y$ una secuencia de 819 pb correspondiente a parte de la región 3' no traducida del ARNm.

\section{Mutagénesis sitio dirigida}

La mutagénesis sitio dirigida para introducir mutaciones específicas en el gen de la proteína p6 $7^{\text {phox }}$ se realizó con el producto comercial GeneEditor $^{\mathrm{TM}}$ in vitro Site-Directed Mutagenesis System (Promega, Madison, WI). Las mutantes obtenidas a partir del cADN de $p 67^{\text {phox }}$ clonado en el vector pVL1393 fueron las siguientes: Sust $\mathrm{G}_{496} \rightarrow \mathrm{A}$, Sust $\mathrm{C}_{985} \rightarrow \mathrm{T}$ y Sust $\mathrm{C}_{1167} \rightarrow \mathrm{A}$ que generaron un cambio en los aminoácidos V166I, $\mathrm{P} 329 \mathrm{~S}$ y $\mathrm{H} 389 \mathrm{Q}$, respectivamente.

\section{Subclonación de las diferentes mutantes de p67 $7^{\text {phox }}$ en el vector pcADN3.1/zeo(+)}

Los cADN de p67 phox tipo silvestre y mutantes obtenidos por mutagénesis sitio dirigida se subclonaron en el vector pcADN3.1/zeo(+) (Invitrogen, Carlsbad, CA) utilizando las enzimas de restricción BamHI y Pstl (Promega). Para la subclonación se realizó una digestión parcial, ya que el cADN de p67 ${ }^{\text {phox }}$ contiene un sitio de corte interno para BamHl en el nucleótido 966 corriente 
arriba del codón de iniciación. En cada uno de los plásmidos obtenidos se verificó la inserción correcta y la presencia del cADN de $p 67^{\text {phox }}$ por medio de una digestión con las enzimas de restricción HindlIIy BamHI. Los nuevos vectores obtenidos se denominaron como pcADN-G ${ }_{496} \rightarrow A$, pcADN-C ${ }_{985} \rightarrow$ T, pcADN-C ${ }_{1167} \rightarrow$ A y pcADN-67WT. Con el fin de verificar la inserción correcta de los diferentes cADN de p6 $7^{\text {phox }}$ clonados en el vector pcADN3.1/zeo(+), así como la presencia de las mutaciones generadas por mutagénesis sitio dirigida, se obtuvo la secuencia del cADN de p6 $7^{\text {phox }}$ en un secuenciador ABI 310 (Perkin Elmer Applied Biosystems, Foster City, CA) con el estuche comercial BigDye Terminator Cycle Sequencing (Perkin Elmer Applied Biosystems), el oligonucleótido universal del promotor de T7 y un oligonucleótido en dirección sentido que forma híbridos con los nucleótidos 813 al 831 del cDNA de $667^{\text {phox }}$.

\section{Transfección de la línea celular COS91/22/47}

Para expresar de manera transitoria las $p 67^{\text {phox }}$ normal y mutante se utilizó la línea celular COS91/ 22/47. Esta línea celular había sido transfectada previamente de manera estable con los cADN de gp91 phox , p22 $2^{\text {phox }}$ y $\mathrm{p} 47^{\text {phox }}$ clonados en los vectores pEF-PGKpac, pEF-PGKneo y pEF-PGKhygro, respectivamente (40-42). Estas células se cultivaron en medio DMEM $/ 10 \%$ suero bovino fetal (SBF) (Hyclone, Logan, UT) con suplemento de 50 unidades $/ \mathrm{ml}$ de penicilina, $50 \mu \mathrm{g} / \mathrm{ml}$ de estreptomicina (Gibco BRLÒ Life Technologies), $0,2 \mathrm{mg} / \mathrm{ml}$ de higromicina (Invitrogen), $1,8 \mathrm{mg} / \mathrm{ml}$ de $\mathrm{G} 418$ y $1 \mu \mathrm{g} / \mathrm{ml}$ de puromicina (Sigma-Aldrich, St. Louis, MO).

Las células COS91/22/47 adherentes se recolectaron de los platos de cultivo por incubación con tripsina/EDTA (Gibco BRLO Life Technologies) durante 5 minutos a $37^{\circ} \mathrm{C}$. La acción de la tripsina se neutralizó por la adición de DMEM/10\% SBF. Posteriormente, las células se precipitaron por centrifugación a $1200 \mathrm{rpm} \mathrm{a} 4^{\circ} \mathrm{C}$ por 5 minutos; se lavaron en PBS frío en hielo, nuevamente se centrifugaron y rápidamente se resuspendieron en PBS a $4^{\circ} \mathrm{C}$.

Con el fin de transfectar transitoriamente la línea celular COS91/22/47 con los vectores que contenían los cADN normal o mutantes de p67 $7^{\text {phox }}$ se utilizó el reactivo Lipofectamine Plus (Gibco $\mathrm{BRL}$ ). Se mezclaron $3 \times 10^{6}$ células COS91/22/47 con $2 \mu \mathrm{g}$ de los respectivos cADN clonados en pcADN3.1/zeo(+). Éstas se analizaron 21 horas después de la transfección como se menciona en los ensayos de Price et al. $(41,42)$.

\section{Análisis de la expresión de la p67phox tipo normal y mutante en células COS91/22/47 transformadas transitoriamente}

Para determinar la expresión de la p67 phox tanto normal como mutantes, se realizó una inmunodetección con un anticuerpo específico contra $\mathrm{p} 67^{\text {phox. A }}$. partir de las células transfectadas, $1 \times 10^{6}$ células se concentraron por centrifugación y, posteriormente, se resuspendieron en el tampón de lisis $(20 \mathrm{mM}$ Tris- $\mathrm{HCl}$, $\mathrm{pH}$ $8,150 \mathrm{mM} \mathrm{NaCl}, 1 \mathrm{mM}$ de EDTA, 1\% de Tritón X$100,20 \mu \mathrm{g} / \mathrm{ml}$ de quimostatina, $2 \mathrm{mM}$ de fluoruro de fenilmetilsulfonil, PMSF-, $1 \mathrm{mM}$ de 4-(2aminoetil) fluoruro de benzenosulfonilo, AEBSFy $10 \mathrm{mM}$ de leupeptina). La concentración de las proteínas totales en el lisado se cuantificó usando el ensayo de proteínas BSA (Pierce, Rockford, IL). Un total de $10 \mu \mathrm{g}$ del lisado total se sometieron a electroforesis en gel SDS-PAGE $12 \%$ seguido por la transferencia a membranas de nitrocelulosa. Las membranas se bloquearon en TBS-T (tampón de tris salino, Tween-20 al $0,5 \%$ ) con leche descremada baja en grasa al $6 \%$ durante 1 hora; luego, se incubaron durante 1 hora con el anticuerpo policlonal de conejo contra p67 $7^{\text {phox }}$ humana donado por David Lambeth (Emory University, Atlanta, GA) diluido 1:5.000 en TBS-T. Posteriormente, se adicionó un anticuerpo monoclonal anti-IgG de conejo producido en ratón, conjugado con peroxidasa de rábano picante diluido en 1:3.000 en TBS-T y se incubó por 1 hora; la reacción antígeno-anticuerpo se visualizó por medio de quimioluminiscencia ECL (Amershan, Arlington Heights, IL). La presencia de las bandas correspondientes a la p6 $7^{\text {phox }}$ se evidenció en película radiográfica X-Omat (Eastman Kodak Co., Rochester, NY).

\section{Cinética de anión superóxido}

Para determinar la actividad funcional del sistema NADPH oxidasa en las células COS91/22/47 transfectadas transitoriamente con los vectores 
que contenían el cADN de p67 phox se evaluó la producción de anión superóxido. Para esto se realizó un ensayo de cinética cuantitativa basada en la reducción del citocromo c, reacción que es inhibible por la superóxido dismutasa. El ensayo se realizó a $37^{\circ} \mathrm{C}$ con un lector de microplatos Thermomax (Molecular Devices, Inc., Sunnyvale, CA) como se ha descrito previamente $(41,43)$. Brevemente, 2,5 $\times 10^{5}$ células provenientes de cada una de las transfecciones se resuspendieron en 250 ul de PBSG (PBS más $0,5 \mathrm{mM}$ de $\mathrm{MgCl}_{2}$, $0,9 \mathrm{mM}$ de $\mathrm{CaCl}_{2}$ y $7,5 \mathrm{mM}$ de dextrosa) que contenía $75 \mu \mathrm{M}$ de citocromo c. Las células se activaron por la adición de $100 \mu \mathrm{M}$ de ácido araquidónico $(\mathrm{AA})$. Por otro lado, una réplica de las muestras se incubó en paralelo con 250 unidades de superóxido dismutasa (SOD). La producción de anión superóxido se cuantificó usando el coeficiente de extinción de $21.1 \mathrm{mM}^{-1} \mathrm{~cm}^{-1}$ para el citocromo C. El índice máximo de generación de anión superóxido por un intervalo de tres minutos se calculó con el programa Softmax, versión 2.0.

\section{Técnica de NBT en placa}

También se evaluó la actividad funcional del sistema NADPH oxidasa por medio de la técnica de NBT en placa. Para la detección de la reducción del azul de tetrazolio (nitroblue tetrazolium, NBT) (Sigma-Aldrich) se procedió de la siguiente manera. EI NBT utilizado se obtuvo del sobrenadante de una solución saturada de NBT en PBSG (PBS más $0,9 \mathrm{mM}$ de $\mathrm{CaCl}_{2}, 0,5 \mathrm{mM} \mathrm{MgCl}$ y $7,5 \mathrm{mM}$ de glucosa). La mezcla para este ensayo contenía $20 \mu \mathrm{l}$ de células COS91/22/47 transfectadas transitoriamente con los vectores que contenían el cADN de $p 67^{\text {phox }}$ tipo normal o mutantes a una concentración de $5 \times 10^{6}$ células $/ \mathrm{ml}, 60 \mu \mathrm{l}$ de PBSG-NBT, más 0,4 $\mu \mathrm{g} / \mathrm{ml}$ de PMA (Sigma-
Aldrich) y se llevó a un volumen final de 300 ul con PBSG. Esta solución se dispensó en un sistema de láminas-cámara de 8 pozos (USA Scientific, Ocala, FL) y se incubó durante 30 minutos a $37^{\circ} \mathrm{C}$. Posteriormente, se aspiró el sobrenadante y las células se dejaron secar a temperatura ambiente durante 15 minutos. Finalmente, las células se fijaron con metanol al $100 \%$ y se colorearon con safranina al $0,2 \%$ durante un minuto. La observación de las láminas y la determinación del porcentaje de células NBT positivas se realizó contando al azar 200 células, en las cuales se identificó la presencia de precipitados azul oscuro (formazán) en el citoplasma celular por medio de un microscopio de luz a un aumento de 400X. Las células que presentaban precipitado de formazán se clasificaron como positivas para el NBT y las que no presentaban este precipitado como negativas.

\section{Resultados}

\section{Obtención de cADNs mutantes p67 phox $y$ subclonación en el vector pcDNA3.1/zeo (+)}

Con el propósito de analizar si las mutaciones que se habían identificado previamente en individuos normales (25) correspondían a polimorfismos genéticos o eran mutaciones que podrían alterar la función de la proteína de 67 kd del sistema NADPH oxidasa, se introdujeron modificaciones en sitios específicos del cADN del gen NCF-2 por medio de mutagénesis sitio dirigida. Las mutaciones generadas están localizadas en diferentes partes del cADN de p67 phox y correspondían a las sustituciones $G_{496} \rightarrow A, C_{985} \rightarrow T$ y $\mathrm{C}_{1167} \rightarrow \mathrm{A}$. La primera de ellas es una transición que predice para una modificación conservadora

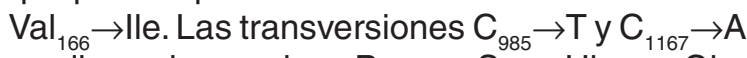
predicen el reemplazo $\mathrm{PrO}_{329} \rightarrow \mathrm{Ser}$ e $\mathrm{His}_{389} \rightarrow \mathrm{Gln}$, respectivamente (figura 1). Posteriormente, estos

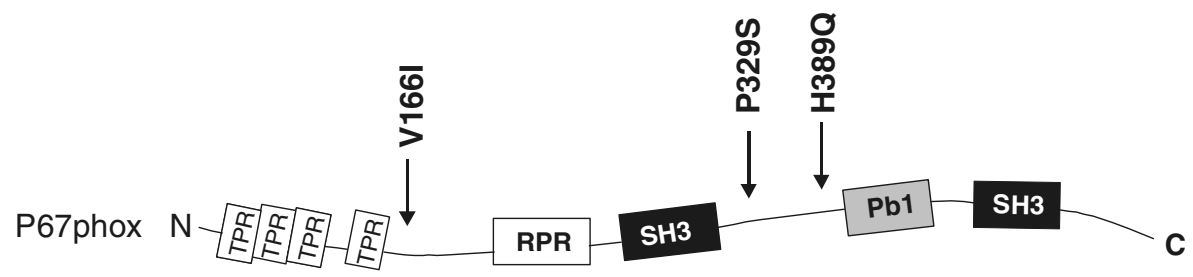

Figura 1. En este esquema se presenta la ubicación de los aminoácidos modificados en la proteína p67phox. El cambio V166I está ubicado entre los dominios TPR y la región rica en prolinas (RPR) de la proteína. Por su parte, las sustituciones P329S y H389Q se encuentran en el extremo carboxilo terminal entre el primer dominio SH3 y el dominio PB1 de la p67 ${ }^{\text {phox. }}$. 


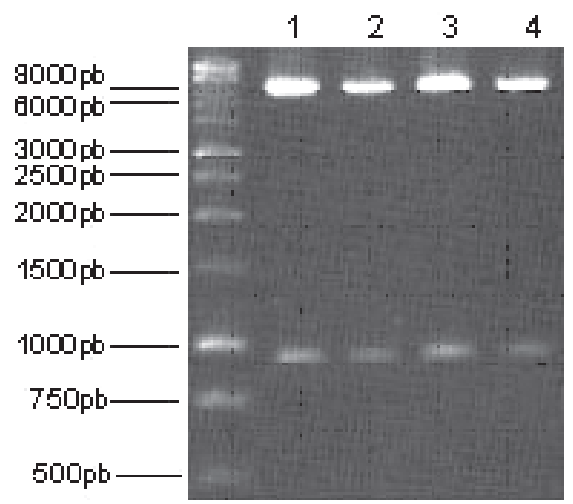

Figura 2. Verificación de la orientación correcta del cADN de $\mathrm{p} 67^{\text {phox }}$ en el vector pcADN3.1/zeo(+). En el gel de agarosa al $1 \%$ coloreado con bromuro de etidio se muestran las digestiones con BamHI y Hindlll de los constructos pcADN$\mathrm{G} 496 \rightarrow \mathrm{A}, \mathrm{pcADN}-\mathrm{C} 985 \rightarrow \mathrm{T}$, pcADN-C1167 $\rightarrow$ A y pcADN$67 \mathrm{WT}$ enumerados del 1 al 4 , respectivamente. La banda de $\sim 966 \mathrm{pb}$ corresponde al fragmento de ADN comprendido entre el sitio HindIII del MCS de pcDNA3.1/zeo(+) y el sitio BamHI que se encuentra 966 pb corriente abajo del codón de iniciación del cADN de p67 phox. La banda de $\sim 6450$ corresponde al resto del inserto de p67 ${ }^{\text {phox }}$ más el vector pcADN3.1/zeo(+). En el lado izquierdo de la fotografía se observa el marcador de longitud de ADN de $1 \mathrm{~kb}$.

CADN se subclonaron en el vector de expresión en mamíferos pcADN3.1/zeo(+) bajo el control del promotor de CMV, lo que permite una eficiente y alta expresión de la proteína recombinante. Los constructos obtenidos fueron pcADN- $G_{496} \rightarrow A$, pcADN-C ${ }_{985} \rightarrow$ T, pcADN-C ${ }_{1167} \rightarrow$ A y pcADN-67WT (tipo normal), los cuales tenían un tamaño de 7415 $\mathrm{pb}$. La orientación correcta del inserto se verificó por medio de digestión con las enzimas BamHl y Hindll que, como se observa en la figura 2, genera un fragmento de $6419 \mathrm{pb}$ y otro de $996 \mathrm{pb}$, lo cual indica la construcción correcta de los nuevos vectores. La verificación de las mutaciones generadas por mutagénesis sitio dirigida se realizó al obtener la secuencia de la región del cADN de p $67^{\text {phox }}$ donde se encontraba la mutación introducida por los respectivos oligonucleótidos (datos no mostrados).

La transfección de los genes modificados permite una expresión adecuada de las proteínas mutantes de $067^{\text {phox }}$ en las células COS91/22/47.

Para confirmar que los cADN que codificaban para la proteína tipo normal y las diferentes mutantes

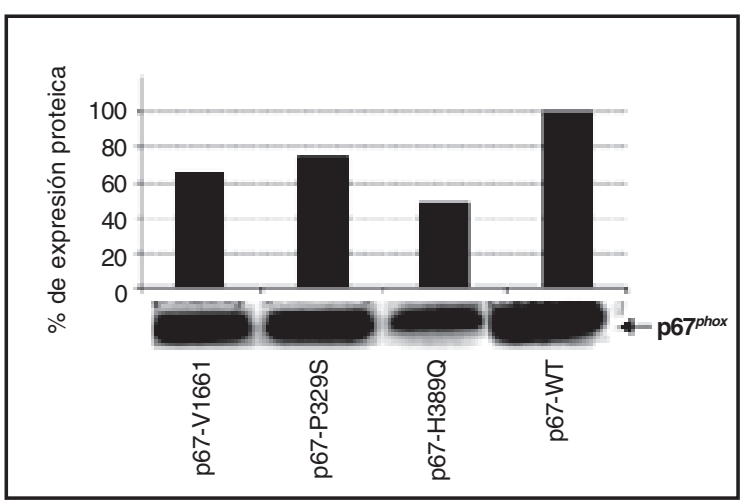

Figura 3. Análisis de la expresión de las proteínas normal y mutantes de p67 $7^{\text {phox }}$ por medio de inmunodetección en las células COS91/22/47. 1 × $10^{6}$ células COS91/22/47 transfectadas transitoriamente con los vectores pcADNG496 $\rightarrow$ A, pcADN-C985®T, pcADN-C1167®A y pcADN$67 W T$ fueron lisadas a las 24 horas postransfección. En la figura se muestran los porcentajes de expresión obtenidos por densitometría comparados con la expresión de la proteína $167^{\text {phox }}$ tipo normal. En la figura se muestra un inmunoblot representativo de cinco análisis realizados.

se expresan en las células COS91/22/47, se realizó un Western blot después de 24 horas de la transformación transitoria (figura 3). Estos resultados muestran que tanto la p67 $7^{\text {phox }}$ normal, como las diferentes mutantes se expresaban en las células COS91/22/47 después de la transfección con los constructos pcADN-G ${ }_{496} \rightarrow A$, pcADN- $\mathrm{C}_{985} \rightarrow \mathrm{T}$, pcADN-C ${ }_{1167} \rightarrow$ A y pcADN-67WT. La expresión de las proteínas mutantes fue entre $47 \%$ y $74 \%$ comparadas con la expresión de la proteína $17^{\text {phox }}$ tipo normal. Esta disminución en la expresión de las proteínas mutantes en las células CoS91/22/47, al compararla con la expresión de la $167^{\text {phox }}$ en células transformadas de manera transitoria con el cADN tipo normal puede deberse a una posible inestabilidad proteica o a una regulación de la expresión intracelular de estas proteínas o, simplemente, es un problema inherente al tipo de transfección transitoria utilizado.

Los polimorfismos de p67phox analizados no afectan la función del sistema NADPH oxidasa.

Para determinar la actividad funcional del sistema NADPH oxidasa en las células COS91/22/47 transformadas transitoriamente con los vectores que contenían el cADN de $p 67^{\text {phox }}$ con los tres 
polimorfismos $\mathrm{G}_{496} \rightarrow \mathrm{A}, \mathrm{C}_{985} \rightarrow \mathrm{T}$ y $\mathrm{C}_{1167} \rightarrow \mathrm{A}$ y con la p6 $7^{\text {phox }}$ normal se evaluó la producción de anión superóxido en un ensayo de cinética cuantitativa basada en la reducción del citocromo c; además,

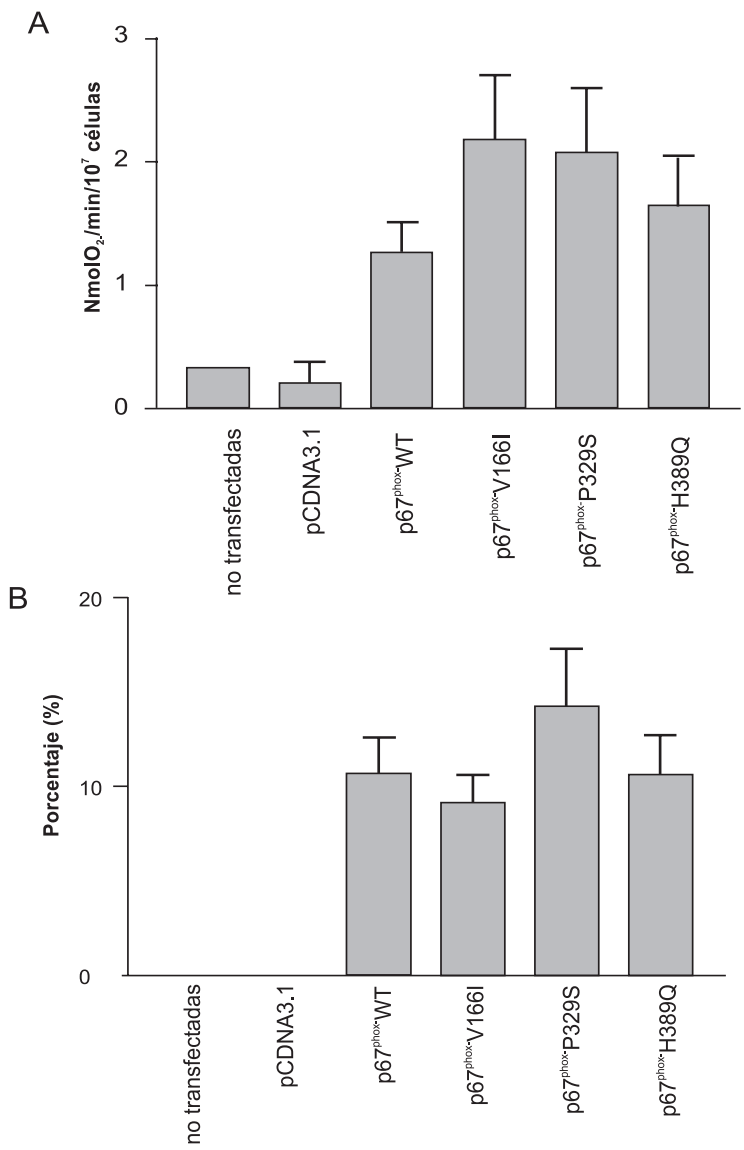

Figura 4. Efecto de los cambios V166I, P329S y H389Q en p $67^{\text {phox }}$ en la producción de $\mathrm{O}_{2}$ comparado con la proteína normal en células COS91/22/47. Las células COS91/22/47 transfectadas transitoriamente con los vectores pcADNG496 $\rightarrow$ A, pcADN-C985 $\rightarrow$ T, pcADN-C1167 $\rightarrow$ A pcADN67WT y pcADN3.1/zeo vacío se analizaron en un ensayo de reducción del ferricitocromo $c$ en células activadas con 100 $\mathrm{mM}$ de ácido araquidónico $(\mathrm{A})$ y por medio de la reducción del NBT con la activación con $0,4 \mu \mathrm{g} / \mathrm{ml}$ de PMA (B). En el ensayo de reducción del ferricitocromo $\mathrm{c}$, los datos representan la media \pm el error estándar (ES), $n=5$. La tasa de producción de anión superóxido no presentó diferencia entre las células que expresaban la p6 $7^{\text {phox }}$ silvestre y las que tenían los cambios V166I, P329S y H389Q en p67 $7^{\text {phox }}$ (prueba t de Student no pareada). En el ensayo de NBT los datos representan la media \pm el error estándar (ES), $n=7$. El porcentaje de reducción de NBT tampoco difirió significativamente entre las células que expresan la p67 phox silvestre y las que expresaban los cambios V166I, P329S y H389Q en p67 phox (prueba t de Student no pareada). se determinó la capacidad que tenían estas células para reducir el azul de tetrazolio por medio de la técnica de NBT en placa. La primera de estas sustituciones determina para una modificación conservadora de la valina 166 por isoleucina $\left(\mathrm{Val}_{166} \rightarrow \mathrm{lle}\right)$. Las células que expresaban esta proteína produjeron anión superóxido a razón de $2,17 \pm 0,52 \mathrm{nmol} / 10^{7}$ células/min $(n=5)$, mientras que el porcentaje de reducción del NBT era de $9,05 \pm 1,57(n=7)$ (figura 4A y 4B). Estos datos no presentaban una diferencia estadísticamente significativa comparada con las células que expresaban la proteína normal donde la producción de anión superóxido fue de 1,26 $\pm 0,24 \mathrm{nmol} / 10^{7}$ células $/ \mathrm{min}(\mathrm{n}=5)$ y el porcentaje de reducción del NBT fue de 10,67 $\pm 1,95(n=7)$. Las células que contenían los cambios $\mathrm{C}_{985} \rightarrow T$ y $\mathrm{C}_{1167} \rightarrow \mathrm{A}$ que predicen el reemplazo de $\mathrm{PrO}_{329} \rightarrow$ Ser y $\mathrm{His}_{389} \rightarrow \mathrm{Gln}$ produjeron $2,07 \pm 0,53 \mathrm{nmol} / 10^{7}$ células $/ \mathrm{min}(n=5)$ y $1,64 \pm 0,40 \mathrm{nmol} / 10^{7}$ células/min $(n=5)$, respectivamente. En el ensayo de NBT, el total de células que trasportaba la transversión $\mathrm{C}_{985} \rightarrow T$ tenía la capacidad de reducir el NBT en un porcentaje de $14,18 \pm 3,12(n=7)$, mientras que las que tenían la transversión $\mathrm{C}_{1167} \rightarrow \mathrm{A}$ fue de $10,58 \pm 2,11(n=7)$. Estos resultados muestran que no hay diferencia significativa si se compara con los resultados obtenidos con la p67 $7^{\text {phox }}$ silvestre $(10,67 \pm 1,95, n=7)$.

\section{Discusión}

En un trabajo previo, nuestro grupo describió la caracterización genética y molecular de varios pacientes con enfermedad granulomatosa crónica autosómica recesiva causada por defectos en la proteína p67 $7^{\text {phox }}$, lo cual permitió identificar una amplia diversidad de mutaciones que afectaban aspectos relacionados con la estructura del gen NCF2 y con la expresión de la p67 $7^{\text {phox }}$ (25). Además, se evidenciaron algunas sustituciones nucleotídicas en sujetos usados como controles. Puesto que la presencia del fenotipo de enfermedad granulomatosa crónica depende de la presencia de ambos alelos mutados, en este caso en el gen NCF-2, no era posible saber si estos cambios correspondían a polimorfismos en este gen o si alguna de ellas era una mutación presente en un individuo portador. Por lo tanto, consideramos importante avanzar en el estudio 
genético y funcional de la $\mathrm{p} 67^{\text {phox }}$ teniendo como modelo las sustituciones encontradas. Basados en estos hallazgos, se generaron por medio de mutagénesis sitio dirigida en el cADN de p67 $7^{\text {phox }}$ los cambios correspondientes con el objetivo de evaluar en un sistema celular su efecto en la activación del sistema NADPH oxidasa.

Para el análisis funcional de las proteínas con mutaciones se utilizaron las células COS91/22/ 47 transfectadas transitoriamente y estimuladas con ácido araquidónico o PMA, lo cual permite determinar la funcionalidad del sistema NADPH oxidasa en células intactas. Esto es importante porque muchas de las características del ensamble del sistema oxidasa no se pueden duplicar en ensayos de sistemas libres de células, pues no reflejan el fenómeno observado en células enteras. Por ejemplo, la fosforilación de p47 $7^{\text {phox }}$ se requiere para la actividad del sistema NADPH oxidasa en neutrófilos (44-46), pero no se requiere para estudios en sistemas libres de células $(1,5,47)$. Por otro lado, las células fagocíticas no son fácilmente transfectables, mientras que el análisis funcional de proteínas mutantes phox en las líneas celulares PBL-985 y linfocitos B transformados con EBV (LB-EBV), las cuales expresan el sistema NADPH oxidasa, tienen limitaciones genéticas y la expresión de los genes transfectados es poco eficiente; además, los linfocitos $B$ tienen bajos niveles de producción de anión superóxido. El sistema NAPDH oxidasa también ha sido reconstituido por transfección en líneas de células hematopoyéticas no fagocíticas K562, las cuales pueden llegar a producir anión superóxido a una taza de $5 \%$ a $15 \%$ comparados con los neutrófilos humanos $(48,49)$.

Los resultados obtenidos en este trabajo demuestran que las sustituciones $\mathrm{Val}_{166} \rightarrow \mathrm{lle}$, $\mathrm{PrO}_{329} \rightarrow$ Ser y His ${ }_{389} \rightarrow \mathrm{Gln}$ no limitaron de manera significativa la producción de anión superóxido en las células COS91/22/47 transfectadas con los cADN que contenían estas mutaciones, comparado con las células transfectadas con el cADN para la proteína tipo silvestre. De acuerdo con estos resultados, dichos cambios en la secuencia del gen NCF-2 pueden ser considerados como polimorfismos genéticos que no afecta la función.
Los polimorfismos genéticos son variaciones comunes que ocurren frecuentemente en cualquier genoma y, en algunos casos, pueden alterar tanto la expresión como la función de un producto genético. Muchos polimorfismos son fenotípicamente benignos en el individuo normal; sin embargo, en un contexto ambiental y genético apropiado, un polimorfismo en una población dada puede actuar como un gen de susceptibilidad y modificar la expresión clínica de una enfermedad (50-55). Por ejemplo, en un estudio reciente se demostró una asociación de complicaciones clínicas en pacientes con enfermedad granulomatosa crónica con la presencia de polimorfismos en genes relacionados con el sistema inmune (50).

Algunos de estos cambios en la secuencia de la p6 $7^{\text {phox }}$ pudieran alterar en cierta forma la correcta expresión o función de esta proteína y, por lo tanto, los individuos portadores de dichos polimorfismos podrían tener una pequeña disminución en la producción de especies reactivas del oxígeno sin llegar a presentar un fenotipo de enfermedad granulomatosa crónica. De esta manera, los portadores de estos cambios podrían aumentar su riesgo genético en asocio con otros polimorfismos para la aparición de enfermedades relacionadas con la disminución de la respuesta inmune innata. Sin embargo, en el sistema celular utilizado en el presente trabajo, las proteínas que portaban estos tres polimorfismos se asociaron con la generación de anión superóxido en cantidades similares a las de la p67 $7^{\text {phox }}$ tipo normal. Esto sugiere que ninguno de los tres polimorfismos está asociado con cambios en la expresión o función de la p6 $7^{\text {phox }}$ que conduzcan a una disminución en la producción de especies reactivas del oxígeno. En el caso del cambio $\mathrm{Val}_{166} \rightarrow$ lle, en el cual ambos son aminoácidos alifáticos y se comportan como hidrófobos, no se esperaba un cambio trascendental en las características funcionales de la proteína, lo que concuerda con los resultados encontrados. En cambio, en la modificación Pro $_{329} \rightarrow$ Ser, se reemplaza un aminoácido que tiene características alifáticas y es hidrófobo por una aminoácido débilmente polar. Como consecuencia de esto, se esperaría un cambio en la actividad del sistema oxidasa; sin embargo, no se encontraron 
diferencias estadísticamente significativas con respecto a la producción de anión superóxido entre las dos proteínas. Posiblemente esto se deba a que el sitio del polimorfismo no se encuentra en un dominio importante de la proteína o a que los cambios de carga generados no producen alteraciones dramáticas en la estructura terciaria de p67 ${ }^{\text {phox }}$. En el último polimorfismo estudiado $\left(\mathrm{His}_{389} \rightarrow \mathrm{Gln}\right.$ ), se cambió un aminoácido básico, fuertemente polar e hidrofílico, por otro polar e hidrofílico. Ambos aminoácidos se encuentran, por lo general, en la superficie exterior de las proteínas interactuando con el medio acuoso, lo que puede estar explicando los resultados obtenidos en los cuales no se observó ninguna diferencia estadísticamente significativa cuando el cADN con el polimorfismo fue transfectado en células Cos91/22/47 y su actividad se comparó con la función de la proteína expresada por el cADN tipo normal de $067^{\text {phox }}$.

En conclusión, en el presente trabajo se determinó el efecto que tienen algunos polimorfismos en el gen de la p67 $7^{\text {phox }}$ sobre la función del sistema NADPH oxidasa usando un sistema celular transgénico no hematopoyético basado en células COS-7. Con este modelo de células completas se pudo determinar que los cambios $\mathrm{Val}_{166} \rightarrow l l e$, $\mathrm{PrO}_{329} \rightarrow$ Ser y $\mathrm{His}_{389} \rightarrow \mathrm{Gln}$ no se asociaban con ninguna alteración que conllevara a una disminución en la actividad del sistema NADPH oxidasa. De esta manera, el modelo celular $\mathrm{COS}^{\text {phox }}$ representa un nuevo sistema de células completas que sirve para estudiar la función del sistema NADPH oxidasa de las células fagocíticas y sus características genéticas.

\section{Agradecimientos}

La realización de este trabajo ha sido posible gracias a la financiación de Colciencias (proyecto 1115-04-12013) y del Comité de Investigaciones de la Universidad de Antioquia. Agradecemos igualmente a Marianne Price por toda su asesoría y por compartir todos sus conocimientos relacionados con el sistema Cos $^{\text {phox }}$.

\section{Referencias}

1. Leto TL. The respiratory burst oxidase. En: Gallin JI, Snyderman R, editors. Inflammation: basic principles and clinical correlates. Third edition. Philadelphia: Lippincott Williams and Wilkins; 1999. p.769-86.

2. Cross AR, Jones OT. Enzymic mechanisms of superoxide production. Biochim Biophys Acta 1991; 1057:281-98.

3. Banfi B, Schrenzel J, Nusse 0 et al. A novel $\mathrm{H}(+)$ conductance in eosinophils: unique characteristics and absence in chronic granulomatous disease. J Exp Med 1999;190:183-94.

4. Taylor WR, Jones DT, Segal AW. A structural model for the nucleotide binding domains of the flavocytochrome b-245 beta-chain. Protein Sci 1993;2: 1675-85.

5. Babior BM. NADPH oxidase: an update. Blood 1999;93: 1464-76.

6. DeLeo FR, Quinn MT. Assembly of the phagocyte NADPH oxidase: molecular interaction of oxidase proteins. J Leukoc Biol 1996;60:677-91.

7. Dinauer MC. Regulation of neutrophil function by Rac GTPases. Curr Opin Hematol 2003;10:8-15.

8. Kitsis E, Weissmann G. The role of the neutrophil in rheumatoid arthritis. Clin Orthop 1991;265:63-72.

9. Boxer LA, Axtell R, Suchard S. The role of the neutrophil in inflammatory diseases of the lung. Blood Cells 1990;16:25-40.

10. Voncken JW, van Schaick H, Kaartinen V et al. Increased neutrophil respiratory burst in bcr-null mutants. Cell 1995;80:719-28.

11. Curnutte JT. Chronic granulomatous disease: the solving of a clinical riddle at the molecular level. Clin Immunol Immunopathol 1993;67:S2-15.

12. Southorn PA, Powis G. Free radicals in medicine. I. Chemical nature and biologic reactions. Mayo Clin Proc 1988;63:381-9.

13. Southorn PA, Powis G. Free radicals in medicine. II. Involvement in human disease. Mayo Clin Proc 1988;63: 390-408.

14. Klebanoff SJ. Oxygen metabolites from phagocytes. En: Gallin Jl, Snyderman R, editors. Inflammation: basic principles and clinical correlates. Third edition. Philadelphia: Lippincott Williams and Wilkins; 1999. p.721-68.

15. Segal BH, Leto TL, Gallin JI, Malech HL, Holland SM. Genetic, biochemical, and clinical features of chronic granulomatous disease. Medicine (Baltimore) 2000;79:170-200.

16. Patino PJ, Perez JE, Lopez JA et al. Molecular analysis of chronic granulomatous disease caused by defects in gp91-phox. Hum Mutat 1999;13:29-37.

17. Rae J, Newburger PE, Dinauer MC, Noack D, Hopkins PJ, Kuruts R, Curnutte JT. X-Linked chronic granulomatous disease: mutations in the CYBB gene 
encoding the gp91-phox component of respiratory-burst oxidase. Am J Hum Genet 1998;62:1320-31.

18. Roos D. The genetic basis of chronic granulomatous disease. Immunol Rev 1994;138:121-57.

19. Ahlin A, De Boer M, Roos D, Leusen J, Smith CI, Sudin U, Rabbani H, Palmblad J, Elinder G. Prevalence, genetics and clinical presentation of chronic granulomatous disease in Sweden. Acta Paediatr 1995; 84:1386-94

20. Tanugi-Cholley LC, Issartel JP, Lunardi J, Freycon F, Morel F, Vignais PV. A mutation located at the $5^{\prime}$ splice junction sequence of intron 3 in the p67phox gene causes the lack of p67phox mRNA in a patient with chronic granulomatous disease. Blood 1995;85: 242-9.

21. Leusen JH, de Klein A, Hilarius PM, Ahlin A, Palmbland J, Edvard Smith, Cl, Diekmann D, Hall A, Verhoeven AJ, Roos D. Disturbed interaction of p21-rac with mutated p67-phox causes chronic granulomatous disease. J Exp Med 1996;184:1243-9.

22. Aoshima M, Nunoi $H$, Shimazu M, Tatsuzawa $O$, Kenney, RT, Kanegasaki S. Two-exon skipping due to a point mutation in p67-phox-deficient chronic granulomatous disease. Blood 1996;88:1841-5.

23. Bonizzato A, Russo MP, Donini M, Dusi S. Identification of a double mutation (D160V-K161E) in the p67phox gene of a chronic granulomatous disease patient. Biochem Biophys Res Commun 1997; 231:861-3.

24. Noack D, Rae J, Cross AR, Muñoz J, Salmen S, Mendoza JA, Rossi N, Curnutte JT, Heyworth PG. Autosomal recessive chronic granulomatous disease caused by novel mutations in NCF-2, the gene encoding the p67-phox component of phagocyte NADPH oxidase. Hum Genet 1999;105:460-7.

25. Patino PJ, Rae J, Noack D, Erickson R, Ding J, García de Olarte D, Curnutte JT. Molecular characterization of autosomal recessive chronic granulomatous disease caused by a defect of the nicotinamide adenine dinucleotide phosphate (reduced form) oxidase component p67-phox. Blood 1999;94: 2505-14

26. Kenney RT, Malech HL, Epstein ND, Roberts RL, Leto TL. Characterization of the p67phox gene: genomic organization and restriction fragment length polymorphism analysis for prenatal diagnosis in chronic granulomatous disease. Blood 1993;82:3739-44.

27. Das AK, Cohen PW, Barford D. The structure of the tetratricopeptide repeats of protein phosphatase 5 : implications for TPR-mediated protein-protein interactions. Embo J 1998;17:1192-9.

28. Diekmann D, Abo A, Johnston C, Segal AW, Hall A. Interaction of Rac with p67phox and regulation of phagocytic NADPH oxidase activity. Science 1994;265: 531-3.
29. Grizot S, Fieschi F, Dagher MC, Pebay-Peyroula E. The active $\mathrm{N}$-terminal region of p67phox. Structure at 1.8 A resolution and biochemical characterizations of the A128V mutant implicated in chronic granulomatous disease. J Biol Chem 2001;276:21627-31.

30. Dang PM, Johnson JL, Babior BM. Binding of nicotinamide adenine dinucleotide phosphate to the tetratricopeptide repeat domains at the $\mathrm{N}$-terminus of p67PHOX, a subunit of the leukocyte nicotinamide adenine dinucleotide phosphate oxidase. Biochemistry 2000;39:3069-75.

31. Koga $H$, Terasawa $H$, Nunoi $H$, Takeshige $K$, Inagaki F, Sumimoto H. Tetratricopeptide repeat (TPR) motifs of p67(phox) participate in interaction with the small GTPase Rac and activation of the phagocyte NADPH oxidase. J Biol Chem 1999;274:25051-60.

32. Lapouge K, Smith SJ, Walker PA, Gamblin SJ, Smerdon SJ, Rittinger K. Structure of the TPR domain of p67phox in complex with Rac.GTP. Mol Cell 2000;6: 899-907.

33. de Mendez I, Garrett MC, Adams AG, Leto TL. Role of p67-phox SH3 domains in assembly of the NADPH oxidase system. J Biol Chem 1994;269:16326-32.

34. Leto TL, Lomax KJ, Volpp BD, Nunoi H, Sechler JMG, Nauseef, WM, Clark RA, Gallin JI, Malech, HL. Cloning of a 67-kD neutrophil oxidase factor with similarity to a noncatalytic region of p60c-src. Science 1990;248:727-30.

35. Leto TL, Garrett MC, Fujii H, Nunoi H. Characterization of neutrophil NADPH oxidase factors p47-phox and p67-phox from recombinant baculoviruses. J Biol Chem 1991;266:19812-8.

36. Zhan S, Vazquez N, Wientjes FB, Budarf ML, Schrock e, Ried T, Green ED, Chanock SJ. Genomic structure, chromosomal localization, start of transcription, and tissue expression of the human p40-phox, a new component of the nicotinamide adenine dinucleotide phosphate-oxidase complex. Blood 1996; 88:2714-21.

37. Fuchs A, Dagher MC, Vignais PV. Mapping the domains of interaction of p40phox with both p47phox and p67phox of the neutrophil oxidase complex using the two-hybrid system. J Biol Chem 1995;270:5695-7.

38. Tsunawaki S, Mizunari $H$, Nagata M, Tatsuzawa $O$, Kuratsuji T. A novel cytosolic component, p40phox, of respiratory burst oxidase associates with p67phox and is absent in patients with chronic granulomatous disease who lack p67phox. Biochem Biophys Res Commun 1994;199:1378-87.

39. Sambrook J, Russell DW. Preparation of cDNA libraries and gene identification. Molecular cloning. A laboratory manual. Vol. 2. New York: Cold Spring Harbor Laboratory Press; 2001. p.11.114.

40. Yu L, Zhen L, Dinauer MC. Biosynthesis of the phagocyte NADPH oxidase cytochrome b558. Role of heme incorporation and heterodimer formation in 
maturation and stability of gp91phox and p22phox subunits. J Biol Chem 1997;272:27288-94.

41. Price MO, Atkinson SJ, Knaus UG, Dinauer MC. Rac activation induces NADPH oxidase activity in transgenic COSphox cells, and the level of superoxide production is exchange factor-dependent. $\mathrm{J}$ Biol Chem 2002;277:19220-8.

42. Price MO, McPhail LC, Lambeth JD, Han $\mathrm{CH}$, Knaus UG, Dinauer MC. Creation of a genetic system for analysis of the phagocyte respiratory burst: high-level reconstitution of the NADPH oxidase in a nonhematopoietic system. Blood 2002;99:2653-61.

43. Zhen L, King AA, Xiao Y, Chanock SJ, Orkin SH, Dinauer MC. Gene targeting of $X$ chromosome-linked chronic granulomatous disease locus in a human myeloid leukemia cell line and rescue by expression of recombinant gp91phox. Proc Natl Acad Sci USA 1993; 90:9832-6.

44. Rotrosen D, Leto TL. Phosphorylation of neutrophil 47-kDa cytosolic oxidase factor. Translocation to membrane is associated with distinct phosphorylation events. J Biol Chem 1990;265:19910-5.

45. Hayakawa T, Suzuki K, Suzuki S, Andrews PC, Babior BM. A possible role for protein phosphorylation in the activation of the respiratory burst in human neutrophils. Evidence from studies with cells from patients with chronic granulomatous disease. J Biol Chem 1986;261:9109-15.

46. Heyworth PG, Badwey JA. Continuous phosphorylation of both the 47 and the $49 \mathrm{kd}$ proteins occurs during superoxide production by neutrophils. Biochim Biophys Acta 1990;1052:299-305.

47. Nauseef WM, McCormick S, Renee J, Leidal KG, Clark RA. Functional domain in an arginine-rich carboxyl-terminal region of p47phox. J Biol Chem 1993; 268:23646-51.

48. de Mendez I, Adams AG, Sokolic RA, Malech HL, Leto TL. Multiple SH3 domain interactions regulate
NADPH oxidase assembly in whole cells. Embo J 1996; 15:1211-20.

49. de Mendez I, Leto TL. Functional reconstitution of the phagocyte NADPH oxidase by transfection of its multiple components in a heterologous system. Blood 1995;85:1104-10.

50. Foster CB, Lehrnbecher T, Mol F, Steinberg SM, Venzon DJ, Walsh TJ, Noack D, Rae J, Winkelstein JA, Curnutte JT, Chanock SJ. Host defense molecule polymorphisms influence the risk for immune-mediated complications in chronic granulomatous disease. J Clin Invest 1998;102:2146-55.

51. Taylor JG, Choi EH, Foster CB, Chanock SJ. Using genetic variation to study human disease. Trends $\mathrm{Mol}$ Med 2001;7:507-12.

52. Levesque MC, Hobbs MR, Anstey NM, Vaughn TN, Chacellor JA, Pole A, Perkins DJ, Misukonis MA, Chanock SJ, Granger DL, Weinberg JB. Nitric oxide synthase type 2 promoter polymorphisms, nitric oxide production, and disease severity in Tanzanian children with malaria. J Infect Dis 1999;180:1994-2002.

53. Rider LG, Artlett CM, Foster CB, Ahmed A, Neeman T, Chanocks SJ, Jiménez, SA, Miller FW, et al. Polymorphisms in the IL-1 receptor antagonist gene VNTR are possible risk factors for juvenile idiopathic inflammatory myopathies. Clin Exp Immunol 2000;121:47-52.

54. Foster CB, Lehrnbecher T, Samuels S, Stein S, Mol F, Metcalf JA, Wyvill K, et al. An IL6 promoter polymorphism is associated with a lifetime risk of development of Kaposi sarcoma in men infected with human immunodeficiency virus. Blood 2000;96:2562-7.

55. Foster CB, Zhu S, Erichsen HC, Lehrnbecher T, Hart ES, Choi E, Stein S, Smith MW, Steinberg SM, et al. Polymorphisms in inflammatory cytokines and Fcgamma receptors in childhood chronic immune thrombocytopenic purpura: a pilot study. Br J Haematol 2001;113:596-9. 